\title{
Effect of Disc Position for Acute Closed Lock of the Temporomandibular Joint
}

\author{
Takashi Uchida $^{1,2 *}$, Takashi Iida ${ }^{1,3}$, Osamu Komiyama ${ }^{1,3}$, Hiroshi Yamamoto ${ }^{4}$, Kayo Kuyama ${ }^{5}$ \\ ${ }^{1}$ Orofacial and Head Pain Clinic, Nihon University Hospital at Matsudo, Chiba, Japan \\ ${ }^{2}$ Department of Oral Diagnostics, Nihon University School of Dentistry at Matsudo, Chiba, Japan \\ ${ }^{3}$ Department of Oral Function and Fixed Prosthodontics, Nihon University School of Dentistry at Matsudo, Chiba, Japan \\ ${ }^{4}$ Department of Oral Surgery, Nihon University School of Dentistry at Matsudo, Chiba, Japan \\ ${ }^{5}$ Department of Pathology, Nihon University School of Dentistry at Matsudo, Chiba, Japan \\ Email: *uchida.takashi@nihon-u.ac.jp
}

How to cite this paper: Uchida, T., Iida, T., Komiyama, O., Yamamoto, H. and Kuyama, K. (2021) Effect of Disc Position for Acute Closed Lock of the Temporomandibular Joint. Open Journal of Stomatology, 11, 297-310.

https://doi.org/10.4236/ojst.2021.118026

Received: July 16, 2021

Accepted: August 17, 2021

Published: August 20, 2021

Copyright $\odot 2021$ by author(s) and Scientific Research Publishing Inc. This work is licensed under the Creative Commons Attribution International License (CC BY 4.0).

http://creativecommons.org/licenses/by/4.0/

(c) (i) Open Access

\begin{abstract}
In this manuscript, the authors have studied the Disc Displacement (DD) status of patients with acute Closed Lock (CL) to determine differences between DD with Reduction (DDwR) and DD without Reduction (DDwoR). Among the acute CL patients who visited our hospital within 2 weeks of the onset of CL, we studied 10 patients whose CL was released (DDwR) and 13 patients whose CL was not released (DDwoR). The DDwoR group was significantly older than the DDwR group. Although the mouth opening distance was significantly greater in the DDwoR group than in the DDwR group, the two groups were identical in the duration of CL. Sagittal MRI images showed no significant differences between the two groups in disc length and disc thickness (anterior band, intermediate zone, and posterior band). Multisection sagittal and coronal images identified lateral DD in 7 of the 10 patients in the DDwR group, although no specific direction of the DD was observed in the DDwoR group. Furthermore, deformation of the medial disc was common in the DDwoR group but uncommon in the DDwR group.
\end{abstract}

\section{Keywords}

Temporomandibular Joint Disorder, Anterior Disc Displacement without Reduction, Closed Lock, Multisection MRI

\section{Introduction}

Closed Lock (CL) caused by Disc Displacement without Reduction (DDwoR) of the Temporomandibular Joint (TMJ) is characterized by various clinical symptoms, including painful TMJ and limited mouth opening. Mandibular Manipu- 
lation (MM), the simplest, fastest, and least costly non-invasive approach, is the accepted course of early treatment for CL [1] because it is readily applied to symptomatic patients. $\mathrm{MM}$ is especially useful in treating patients with early DDwoR (short duration CL) [2]. CL duration is the most important factor in determining successful outcomes for MM [3]. Shorter CL duration is often associated with higher rates of MM success [1]. However, favorable MM outcomes were not achieved in certain patients despite the early implementation of $M M$, and the relationship between CL and MM outcomes remains unclear.

Some reports suggest that the findings of imaging studies are not always consistent with clinical symptoms in real-world settings [4]. Diagnoses based on Magnetic Resonance Imaging (MRI) in patients with Temporomandibular Joint Disorder (TMD) tend to more closely correlate with clinical symptoms [5]; thus, accurately ascertaining the complete condition of disc position is crucial for the prognosis and evaluation of therapeutic outcomes [6]. In particular, previous studies have often focused on the analysis of disc deviation on MRI sagittal planes [7] [8] [9]. Few studies have investigated Disc Displacement (DD) in other directions. Because not just anterior but lateral and posterior DD can occur, the findings on multisection images (combined sagittal and coronal planes) need to be analyzed to accurately ascertain the DD direction [10].

Our study investigated differences in the direction of DD based on the multisection MRI analysis of the sagittal and coronal planes. The goal was to determine differences in treatment outcomes, including MM, for unlocking in patients with early (acute) Closed Lock (CL).

\section{Subjects and Method}

\subsection{Patient Sample (Table 1)}

We extracted 245 patients who had visited the Orofacial and Head Pain Clinic, Nihon University Hospital at Matsudo with the chief complaint of temporomandibular dysfunction from January 2016 to December 2020 and who were subsequently diagnosed with TMD based on MRI findings after excluding patients with a TMJ fracture, cyst, tumor, or odontogenic inflammation such as osteomyelitis that could affect the TMJ. Twenty-three patients ( 3 men, 20 women; median age of 26 years [ $32.6 \pm 16.5$ years]) who visited our hospital within 2 weeks after the occurrence of clinical symptoms for CL associated with TMD and were examined by MRI within 1 month after the onset of CL were defined as suffering

Table 1. Subjects occupation.

\begin{tabular}{ccc}
\hline & Female $(\mathrm{n}=\mathbf{2 0})$ & Male $(\mathbf{n}=\mathbf{3})$ \\
\hline High school student & 3 & 1 \\
Univercity student & 5 & 1 \\
Society & 7 & 1 \\
Housewife & 5 & \\
\hline
\end{tabular}


from acute CL and included in the study. The occupations of the subjects are shown in Table 1.

We applied the following exclusion criteria: 1) the patient did not undergo MRI within 4 weeks following the occurrence of symptoms, despite a diagnosis of CL associated with TMD; 2) those with a history of trauma involving the TMJ; 3) those with a history of psychiatric disease, visiting a psychiatric physician, or taking psychotropic drugs; 4) the patient did not provide informed consent after the study was explained.

Patients were treated by specialists qualified by the Japanese Society for Temporomandibular Joint. MM was administered without local anesthesia on first presentation to release CL. A rapid increase in painless mouth opening range without the presence of clear TMJ clicking on the symptomatic side or mandibular deviation at the time of mouth opening was defined as a clinical release of CL [11]. If CL release could not be achieved on the first presentation, patients were instructed to wear a pivoting appliance made of cold-cured resin [12] at night and perform passive exercises [13]; on a later date, the presence/absence of CL release was confirmed. The patients were divided into two group: a group of 13 patients in whom CL was not achieved (DDwoR) and a group of 10 patients in whom CL was achieved (DDwR). In 6 of 10 patients in the DDwR group, CL release was achieved with MM on the first presentation, after which MRI was performed. In the other 4 patients, no reduction was observed at the time of the MRI, but CL release was subsequently clinically confirmed. In one patient, CL release was achieved with MM on the first presentation, but the CL recurred and could not be resolved; this patient was excluded from the study. Twenty-three patients were followed up for 6 months after the diagnosis of DDwoR or DDwR by MRI to check the progress.

\subsection{Image Assessment}

MRI findings obtained for analysis on the central slices of sagittal plane included disc length and disc thickness (anterior band, intermediate zone, posterior band).

\section{Disc position}

The classification of Litko-Rolaetu et al. [10] proposes 10 disc positions, shown below, based on MRI findings for the multisection sagittal and coronal planes.

\section{Disc shape}

The system of Almăşan et al. [14] proposes 5 disc shape types, as shown below, based on evaluations of MRI findings for multisection sagittal planes.

Our MRI findings were evaluated by two observers with more than 10 years' experience in upper TMD treatment and the maxillofacial diagnosis. These observers were blinded so that they did not know whether diagnostic images belonged to the DDwR or DDwoR groups when making their evaluations. In cases of discordant evaluations, the two observers discussed the findings until an agreement was reached (Figure 1, Figure 2). 

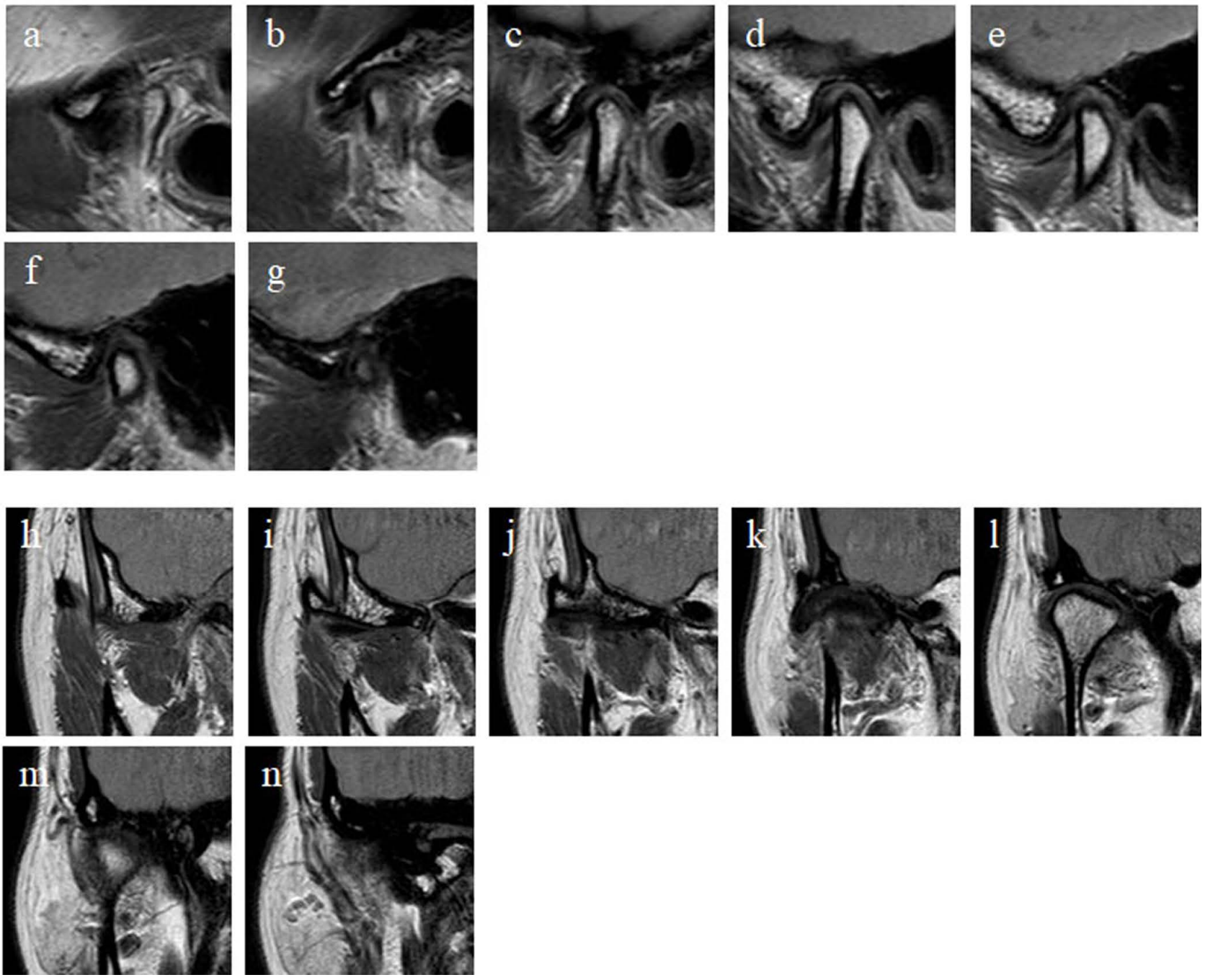

Figure 1. MRI of DDwR in closed mouth position. (a)-(g): Oblique sagittal slices (a to g mean lateral to medial); (h)-(n): Oblique coronal slices (h to $\mathrm{n}$ mean anterior to posterior). In (a), only the disc is observed, but in ( $\mathrm{g}$ ), the disc is not observed and only the condyle is observed. The lateral displaced disc is observed in ( $\mathrm{j})$ and $(\mathrm{k})$.
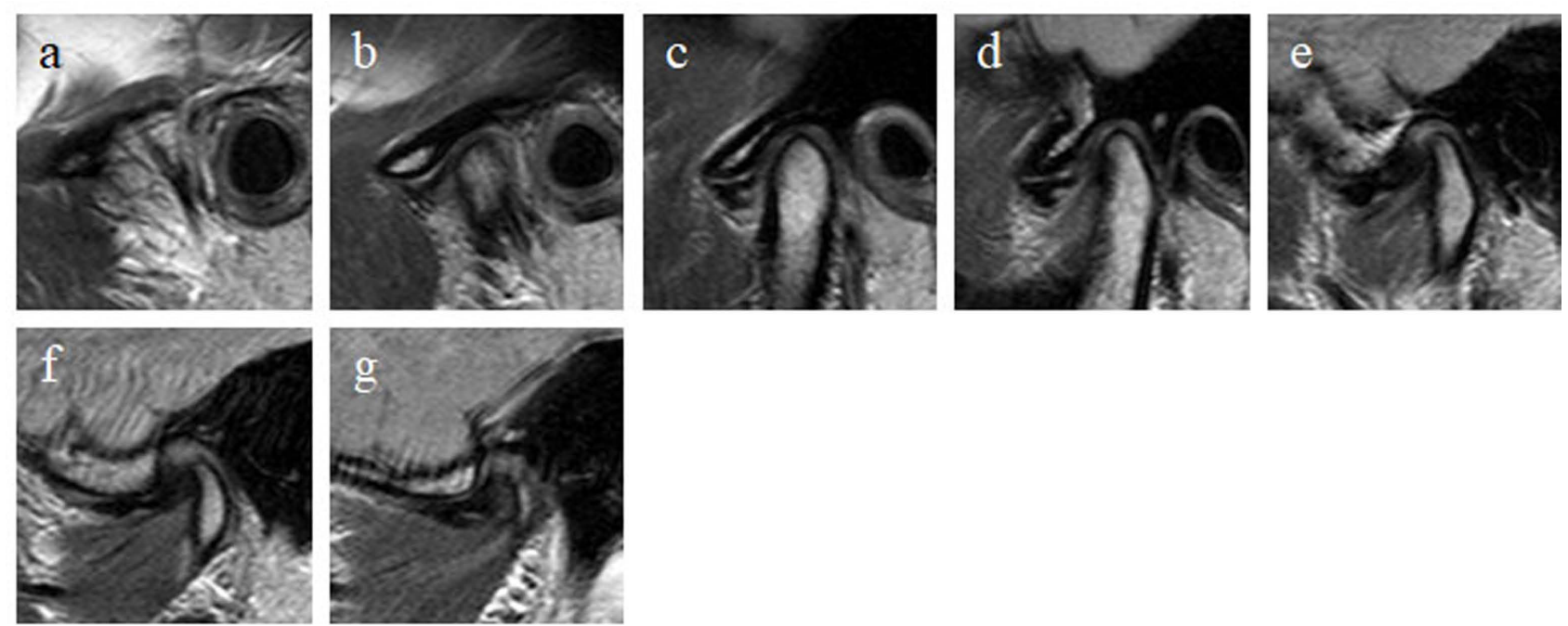

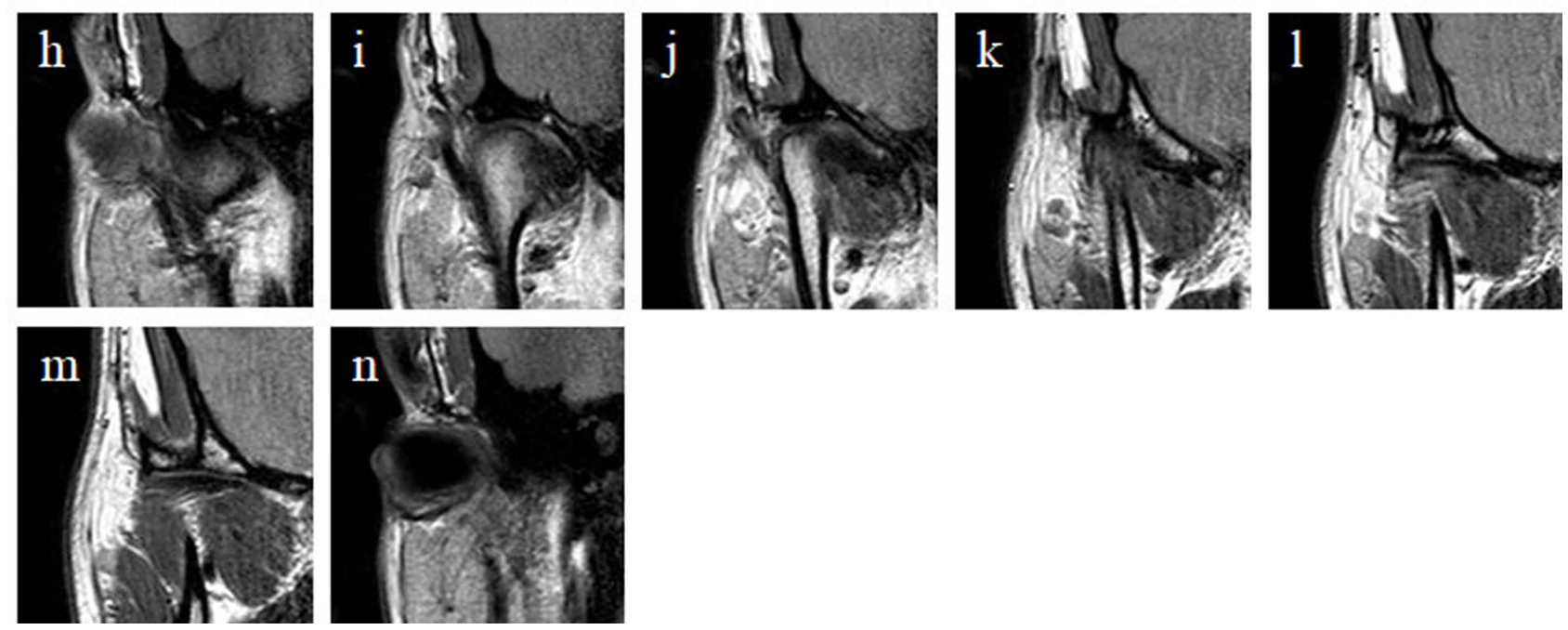

Figure 2. MRI of DDwoR in closed mouth position. (a)-(g): Oblique sagittal slices ((a) to (g) mean lateral to medial). (h)-(n): Oblique coronal slices $(\mathrm{h})$ to $(\mathrm{n})$ mean anterior to posterior). The condyle is not observed at $(\mathrm{g})$, but only the deformed disc is observed. The medial displaced disc is observed in (i), (j), and (k).

\subsection{MRI Examination}

All MRI studies of the TMJ was performed using a 1.5-Tesla unit (Intera Achieva 1.5T; Philips Medical Systems, Best, Netherlands) with a split head coil. All subjects were placed into the standard head coil with fixation devices on both sides. The MRI protocol included proton density fast spin echo sagittal oblique images with the closed and open mouth position, coronal oblique images with the closed mouth position (TR, $1500 \mathrm{~ms}$; TE, $30 \mathrm{~ms}$; FOV, $159 \times 100 \mathrm{~mm}$; and matrix, 256 $\times 256$ ); Coronal oblique slices were placed parallel to the long axis of the mandibular condyles. Sagittal oblique slices were placed perpendicular to the long axis of the mandibular condyles.

\subsection{Statistical Analysis}

We compared patient age, the duration of CL, the mouth opening distance, disc length, and disc thickness between the two groups using the Mann-Whitney $U$ test. We applied Pearson's Chi-square test with Yates' continuity correction to compare disc position distributions between the groups. A value of $p<0.05$ was considered significant.

\section{Results}

\subsection{Age (Table 2)}

Patients in the DDwoR group were significantly older than in the DDwR group $(30.0 \pm 16.8$ years [median \pm quartile deviation] vs. $18.5 \pm 10.7$ years $)(\mathrm{p}=$ $0.030)$.

\subsection{Duration of Closed Lock (Table 2)}

We found no significant difference in CL duration between the DDwoR group 
(5.0 \pm 3.3 days) and DDwR group ( $3.5 \pm 6$ days $)(\mathrm{p}=0.605)$.

\subsection{Mouth Opening Range (Table 2)}

The mouth opening range was $24.0 \pm 1.8 \mathrm{~mm}$ in the DDwoR group and $22.5 \pm$ $2.12 \mathrm{~mm}$ in the DDwR group-significantly greater in the former than in the latter $(\mathrm{p}=0.036)$.

\subsection{Disc}

\subsubsection{Largest Disc Dimension (Table 3)}

\section{1) Disc length}

Disc length on the sagittal images was $9.6 \pm 0.9 \mathrm{~mm}$ in the DDwoR group and $9.6 \pm 1.1 \mathrm{~mm}$ in the DDwR group. We found no significant difference between the groups.

\section{2) Disc thickness (Table 3)}

The maximum disc thickness was $3.6 \pm 0.5 \mathrm{~mm}$ in the DDwoR group and 3.5 $\pm 0.7 \mathrm{~mm}$ in the $\mathrm{DDwR}$ group for the anterior band; $1.5 \pm 0.3 \mathrm{~mm}$ in the DDwoR group and $1.4 \pm 0.2 \mathrm{~mm}$ in the DDwR group for the intermediate zone; and $3.6 \pm 0.8 \mathrm{~mm}$ in the DDwoR group and $3.5 \pm 0.7 \mathrm{~mm}$ in the DDwR group for the posterior band. These variables did not differ significantly between the two groups.

\subsubsection{Distribution of Disc Position (Table 4)}

The DDwo group included complete anterior DD in 3 patients, partial anterior $\mathrm{DD}$ in the lateral part in 1 patient, complete anterolateral DD in 4 patients, and complete anteromedial DD in 5 patients. In the DDwR group, 2 patients had

Table 2. Comparison of subject's age, duration of closed lock, and mouth opening range.

\begin{tabular}{cccc}
\hline & $\begin{array}{c}\text { DDwoR ( }=13) \\
\text { (Median } \pm \text { Quartile } \\
\text { deviation) }\end{array}$ & $\begin{array}{c}\text { DDwR }(\mathbf{n}=10) \\
\text { (Median } \pm \text { Quartile } \\
\text { deviation) }\end{array}$ & \\
\hline Age (year) & $30.0 \pm 16.8$ & $18.5 \pm 10.8^{*}$ & $(\mathrm{p}=0.030)$ \\
Duration of closed lock (day) & $5.0 \pm 3.3$ & $3.5 \pm 6.0$ & $(\mathrm{p}=0.605)$ \\
Mouth opening range (mm) & $24.0 \pm 1.8$ & $22.5 \pm 2.1^{*}$ & $(\mathrm{p}=0.036)$ \\
\hline
\end{tabular}

*Significant difference by Mann-Whitney U test.

Table 3. Disk dimensions in closed mouth position analysed in sagittal plane.

\begin{tabular}{cccc}
\hline & & $\begin{array}{c}\text { DDwoR }(\mathrm{n}=13) \\
\text { (Median } \pm \text { Quartile } \\
\text { deviation) }\end{array}$ & $\begin{array}{c}\text { DDwR }(\mathrm{n}=10) \\
\text { (Median } \pm \text { Quartile } \\
\text { deviation) }\end{array}$ \\
\hline Maximum disk length $(\mathrm{mm})$ & $9.6 \pm 0.9$ & $9.6 \pm 1.1$ \\
& Anterior band $(\mathrm{mm})$ & $3.6 \pm 0.5$ & $3.7 \pm 1.5$ \\
Largest disk thickness & Intermediate zone $(\mathrm{mm})$ & $1.5 \pm 0.3$ & $1.4 \pm 0.2$ \\
& Posterior band $(\mathrm{mm})$ & $3.6 \pm 0.8$ & $3.5 \pm 0.7$ \\
\hline
\end{tabular}

No Significant difference by Mann-Whitney U test. 
complete anterior DD, 7 patients had complete anterolateral DD, and 1 patient had lateral DD. These differences in the distribution of disc position between the two groups $(p=0.495)$ were not significant.

\subsubsection{Distribution of Disc Shape (Table 5)}

In the DDwoR group, we observed various disc shapes on the medial side, including even thickness in 2 patients, posterior band enlargement in 2 patients, folding in 5 patients, and convex in 4 patients. However, in the DDwR group, we observed biconcave (normal shape) in 7 patients. This was more common than posterior band enlargement ( 2 patients) and folding ( 1 patient). Thus, the distribution of disc shape differed significantly between the two groups $(\mathrm{p}=0.047)$.

The disc shape on the lateral side in the DDwoR group was biconcave in $1 \mathrm{pa}-$ tient, even thickness in 2 patients, posterior band enlargement in 3 patients, folding in 6 patients, and convex in 1 patient, exhibiting various disc shapes on the

Table 4. Distribution of disc position in closed mouth position multisectionally analysed in sagittal and coronal plane according to category.

\begin{tabular}{llcc}
\hline & & DDwoR $(\mathrm{n}=13)$ & DDwR $(\mathrm{n}=10)$ \\
\hline a & Normal superior & 3 & 2 \\
b & Complete anterior & 1 & \\
c & Partial anterior in the lateral part & & \\
d & Partial anterior in the medial part & & \\
e & Partial anterolateral, & & \\
f & Partial anteromedial & 4 & \\
g & Complete anterolateral & 5 & \\
h & Complete anteromedial & & \\
i & Lateral & & \\
j & Medial & & \\
& & & \\
\end{tabular}

No Significant difference by Pearson's chi-square test with Yates' continuity correction.

Table 5. Distribution of disc shape in closed mouth position multisectionally analysed in sagittal and coronal plane according to category.

\begin{tabular}{llcccc}
\hline & & \multicolumn{2}{c}{ Medial } & \multicolumn{2}{c}{ Lateral } \\
\cline { 3 - 5 } & & DDwoR & DDwR & DDwoR & DDwR \\
\hline a & Biconcave & & 7 & 1 & 1 \\
b & Even thickness & 2 & 2 & 1 \\
c & Posterior band enlargement & 2 & 3 & 7 \\
d $\quad$ Folding & 5 & 1 & 6 & 1 \\
e $\quad$ Convex & 4 & 1 & $(\mathrm{p}=0.603)$ \\
\hline
\end{tabular}

*Significant difference by Pearson's chi-square test with Yates' continuity correction. 
lateral side, as on the medial side. In the DDwR group, we observed biconcave disc shape in 1 patient, posterior band enlargement in 1 patient, folding in 7 patients, and convex in 1 patient. As in the DDwoR group, the folding shape was the most common in the DDwR group. We found no significant differences in disc shape distributions between the two groups $(p=0.603)$.

\subsubsection{Joint Effusion}

Joint effusion was observed in the temporomandibular joint on the symptomatic side in Twenty-three patients.

\section{Discussion}

Both DDwR and DDwoR are reported to increase the risk of degenerative changes in the mandibular condyle [15]. However, even when DD occurred, it appears beneficial to retain DDwR to maintain a normal condylar surface, bone marrow, and joint effusion [9]. Furthermore, certain biochemical evidence suggests that patients with chronic CL are prone to develop osteoarthritic degeneration [16] [17]. Patients with DDwoR are reported to at 4 -fold increased risk of exhibiting degenerative change than those with joints with normal disc position [18]. Joints with DDwoR do not always progress to osteoarthritic degeneration, but the condition of DDwoR may become chronic and persist. For this reason, it is considered necessary to treat CL (especially in the acute phase) with the aim of producing the unlock condition. Thus, in our clinic, we performed MM in the early phase for patients diagnosed with CL. One report argues that there is insufficient evidence to support the benefit of MM [19]. However, MM is useful as a simple, fast, non-invasive initial treatment of acute CL on first presentation [2]. In particular, since invasive interventions should be avoided as the initial CL management [20], we seek to use unlock MM to release CL to avoid invasive interventions on the patient's first presentation. Our approach resulted in CL release in 6 of 23 patients in this study. Arthrocentesis is sometimes used as the first low invasive surgical intervention for the treatment of slightly movable TMJ associated with pain, thereby reducing pain and TMJ disorders more rapidly than conventional non-surgical procedures [21]. However, it is undesirable to perform invasive procedures in the early phase of patient management. In addition, it is difficult to obtain informed consent from patients for this particular treatment. In our clinic, we use MM without local anesthesia for the upper joint cavity in treatment for the early phase of CL.

If $\mathrm{CL}$ is not released on first presentation, we prepare a pivot sprint made of cold-cured resin on that day and instruct patients to wear the device at night. With respect to pivot sprints, Seedorf et al. [22] report no distractive effect on the TMJ. In contrast, Yilidix et al. [23] report that a pivot sprint has distractive effects on unilateral DD without reduction. Despite scant evidence for the results of pivot sprints, in our clinic, we instruct patients to wear a pivot sprint at night, in addition to performing passive exercises, based on the prospects of the dis- 
tractive effects on TMJ. In 4 of the present 17 patients in whom CL release was not achieved on first presentation, CL release was confirmed in the subsequent days.

The duration of the lock is the most important factor in MM success. In this study, we restricted the duration of lock (i.e., the time up to the MRI) to 4 weeks, based on findings from other studies [2] [24] to investigate patients with acute CL. Thus, the interval between CL onset and the visit to our hospital did not differ between the DDwR and DDwoR groups. Potential factors influencing the success of other treatments include patient age and duration of prior CL [25]. In older patients, deformation of the disc may progress in a chronic and complicated manner, making it difficult to achieve disc reduction [1]. In our study, patients in the DDwoR group were significantly older than in the DDwR group. However, we did not examine the time elapsed from when TMJ clicking occurred. Future research is required to address the duration of subjective symptoms that suggest DD, as well as age, to determine the success of MM. The mouth opening distance was significantly greater in the DDwoR group than in the DDwR group. One mechanism whereby acute DDwoR progresses to chronic phase may be that disc deviation obstructs the forward condylar translation, restricting mouth opening. In the subsequent chronic phase, whenever the patient attempts to open the mouth widely, the disc gradually moves forward and disc deformation progresses, creating a larger space for the forward movement of the mandibular condyle. The mouth opening range then grows over time [26].

In our study, it is possible that patients with DDwoR, after they become aware of their limited ability to open their mouth, made significant efforts to open their mouth before the visit to our hospital, resulting in progressive displacement and deformation of the disc and a subsequent increase in the movability of the mandibular condyle. Thus, we also investigated disc in terms of the disc length and the maximum thickness in the posterior band, intermediate zone, and anterior band [27] using the central slice on the MRI sagittal images, as reported in a previous study [14], to determine differences in these variables between the DDwR group and the DDwoR group. The ongoing disc deformation in cases of CL is believed to shorten the disc and affect the thickness of the posterior band. Our study found no significant differences between the two groups in the changes in disc findings based on sagittal images. The study results suggest that changes in disc findings on sagittal images do not progress rapidly in the acute phase of CL, but are visualized in association with the progression of disc deformation in the chronic phase of CL.

Various combination of DD types are visualized on MRI sagittal images-not just anterior, but posterior, medial, and lateral DD [28]. MRI disc findings must be evaluated on combined multisection sagittal and coronal images to determine changes. In this study, we ascertained the disc position on the combined sagittal and coronal images based on the classification proposed by Litko-Rola et al. [10]. Of 23 cases of TMJ, only 5 joints showed anterior DD not associated with side- 
ways DD. Sideways DD is commonly observed with TMJ; the direction of sideways DD was medial for 5 joints; lateral for 13 joints, which was most common. The conventional and most common type of DD is anteromedial DD [29]. However, disc deviation in the anterolateral direction has recently been shown to be somewhat more common than the previously reported anteromedial direction [30]. Ikeda et al. [31] state that such discrepancies in study results may be attributable to differences in study methodologies. In our study, lateral DD was more common than other types of DD, a finding consistent with the recently reported anterolateral direction.

In comparing types of sideways DD between the DDwR group and the DDwoR group, we found that anterior DD and the associated lateral DD were common; we also observed either medial or lateral DD at the same frequency in the DDwoR group. In the DDwR group, 8 of 10 joints showed lateral DD. This suggests that lateral DD is more common in the DDwR group (Figure 1), although we observed no significant differences in the distribution of DD between the two groups.Comparing MRI findings in the groups of anterior DD with reduction and without reduction, Sener et al. [32] report that medial DD is more common in the group of anterior DDwoR (Figure 2). The explanation they offer is that their study included patients with acute phase anterior DD. Our findings of higher rates of medial DD in the DDwoR group than in the DDwR group are consistent with their findings.

However, another study has shown that the lateral DD is more likely to occur in the early phase of DD [31]. A study by Tasaki et al. [33] of volunteers with asymptomatic DD indicates that the most common type is rotational anterolateral DD. Our previous study [34] also showed that partial anterolateral DD is the most common. We speculate that lateral DD is the direction unlikely to hamper condylar movement. Intermittent CL is also deemed unlikely to cause disturbances in mandibular condyle movement. The anterior DD may be mainly involved in the occurrence of intermittent CL because sideways DD lacks specific features [35]. The results of this study suggest that the pathology of DD may differ from that of intermittent CL for DDwR.

Bringing the mandible to the contralateral side during the MM of CL has been shown to permit contralateral movements that allow easier movement of the condyle [1]. Thus, if lateral DD has occurred, CL may be released more readily by inducing the lateral DD to the contralateral side, which does not hamper the movement of the disc. Since no local anesthesia (used for arthrocentesis) is administered for manual manipulation, whether or not unlock is successfully achieved by manual manipulation depends on the pain sensitivity of the patient. In cases of medial DD, inducing movement to the contralateral side hampers the disc movement associated with the medial DD, resulting in severe pain; thus, patients are reluctant to open their mouth. As a result, the deformation of the medial disc may also occur.

In cases of disc deformation, our study found that the medial side in the 
DDwoR group deviates from the medial pole of the mandibular condyle; thus, we often observed marked deformities in shape on the medial side, including folding and convex. In contrast, in the DDwR group, since the lateral side deviates from the lateral pole, folding was observed most commonly. When the deformation becomes severe on the medial or lateral side corresponding to the direction of $\mathrm{DD}$, disc movement is unlikely to occur on the medial side from the inner surface of the glenoid fossa of the temporal bone, which readily hampers the sliding of the mandibular condyle. However, since the lateral side of the TMJ, unlike the medial surface, is not bone tissue, there is a space for movement to the lateral side associated with sliding of the mandibular condyle. This is unlikely to hamper mandibular movement, potentially contributing to easy DD reduction. Since the manual manipulation administered in this study was performed without local anesthesia, we must account for differences in patient pain sensitivity. Our study suggests that impaired movement of the mandibular condyle associated with DD is more affected on the medial side than on the lateral side. Due to small sample size (23 patients), more extensive and long-term evaluations of DDwR and DDwoR are needed for larger groups.

The study results show that disc deformation is marked across the entire disc from the medial to lateral side in the DDwoR group, while disc deformation is marked on the lateral side but not on the medial side in the DDwR group. In addition, it is unknown whether disc deformation in the medial direction had already occurred on first presentation or occurred as a consequence of MM. According to Ikeda et al. [31], medial DD is generally associated with more extensive DD. Future studies should focus on the mechanism whereby medial DD occurs; that is, studies should seek to determine whether partial anterior DD occurs on the lateral side, lateral DD causes greater disc deformation, and CL ultimately leads to the medial side; or whether medial DD in the presence of CL in which reduction is difficult occurs in the early phase of DD.

\section{Conclusion}

Based on the results of this study, we suggest applying a combination of multi-section sagittal and coronal images to evaluate DD on MRI in patients with TMD. Therapeutic outcomes for unlocking release are likely to be favorable for lateral DD but unfavorable for medial DD in patients with acute CL. The results suggest that the effects on the movement of the mandibular condyle differ significantly depending on whether the sideways DD is on the medial or lateral side.

\section{Conflicts of Interest}

The authors declare no conflicts of interest regarding the publication of this paper.

\section{References}

[1] Okeson, J.S. (2008) Treatment of Temporomandibular Joint Disorders. In: Okeson, J.S., Ed., Management of Temporomandibular Disorders and Occlusion, 6th Edition, 
Mosby, Inc., St. Louis, 405-453.

[2] Al-Baghdadi, M., Durham, J. and Steele, J. (2014) Timing Interventions in Relation to Temporomandibular Joint Closed Lock Duration: A Systematic Review of "Locking Duration". Journal of Oral Rehabilitation, 41, 24-58. https://doi.org/10.1111/joor.12126

[3] Murakami, K., Hosaka, H., Moriya, Y., Segami, N. and Iizuka, T. (1995) Short-Term Treatment Outcome Study for the Management of Temporomandibular Joint Closed Lock. A Comparison of Arthrocentesis to Nonsurgical Therapy and Arthroscopic Lysis and Lavage. Oral Surgery, Oral Medicine, Oral Pathology and Oral Radiology, 80, 253 257. https://doi.org/10.1016/S1079-2104(05)80379-8

[4] Eriksen, E.S., Hellem, S., Skartveit, L., Brun, J.G., Bøe, O.E., et al. (2020) Temporomandibular Joint Pain and Associated Magnetic Resonance Findings: A Retrospective Study with a Control Group. Acta Radiologica Open, 30.

https://doi.org/10.1177/2058460120938738

[5] Jeon, K.J., Lee, C., Choi, Y.J. and Han, S.S. (2021) Analysis of Three-Dimensional Imaging Findings and Clinical Symptoms in Patients with Temporomandibular Joint Disorders. Quantitative Imaging in Medicine and Surgery, 11, 1921-1931. https://doi.org/10.21037/qims-20-857

[6] Zhang, S.Y., Liu, X.M., Yang, C., Cai, X.Y., Chen, M.J., et al. (2009) New Arthroscopic Disc Repositioning and Suturing Technique for Treating Internal Derangement of the Temporomandibular Joint: Part II-Magnetic Resonance Imaging Evaluation. Journal of Oral and Maxillofacial Surgery, 68, 1813-1817.

https://doi.org/10.1016/j.joms.2009.08.012

[7] Park, J.W., Song, H.H., Roh, H.S., Kim, Y.K. and Lee, J.Y. (2011) Correlation between Clinical Diagnosis Based on RDC/TMD and MRI Findings of TMJ Internal Derangement. Journal of Oral and Maxillofacial Surgery, 41, 103-108. https://doi.org/10.1016/j.ijom.2011.09.010

[8] Amaral, R.O., Damasceno, N.N., de Souza, L.A. and Devito, K.L. (2013) Magnetic Resonance Images of Patients with Temporomandibular Disorders: Prevalence and Correlation between Disk Morphology and Displacement. European Journal of Radiology, 82, 990-994. https://doi.org/10.1016/j.ejrad.2013.01.002

[9] Matsubara, R., Yanagi, Y., Oki, K., Hisatomi, M., Santos, K.C., et al. (2018) Assessment of MRI Findings and Clinical Symptoms in Patients with Temporomandibular Joint Disorders. Dentomaxillofacial Radiology, 47, 4. https://doi.org/10.1259/dmfr.20170412

[10] Litko-Rola, M., Szkutnik, J. and Różyło-Kalinowska, I. (2020) The Importance of Multisection Sagittal and Coronal Magnetic Resonance Imaging Evaluation in the Assessment of Temporomandibular Joint Disc Position. Clinical Oral Investigations, 25, 159 168. https://doi.org/10.1007/s00784-020-03347-9

[11] Lei, J., Yap, A.U., Liu, M.Q. and Fu, K.Y. (2019) Condylar Repair and Regeneration in Adolescents/Young Adults with Early-Stage Degenerative Temporomandibular Joint Disease: A Randomised Controlled Study. Journal of Oral Rehabilitation, 46, 704-714. https://doi.org/10.1111/joor.12805

[12] Okeson, J.S. (2008) Occlusal Appliance Therapy. In: Okeson, J.S., Ed., Management of Temporomandibular Disorders and Occlusion, 6th Edition, Mosby, Inc., St. Louis, 468-497.

[13] Okeson, J.S. (2008) General Considerations in the Treatment of Temporomandibular Disorders. In: Okeson, J.S., Ed., Management of Temporomandibular Disorders and Occlusion, 6th Edition, Mosby, Inc., St. Louis, 334-375.

[14] Almăşan, O.C., Hedeşiu, M., Băciuț, G., Leucuța, D.C. and Băciuț, M. (2013) Disk and 
Joint Morphology Variations on Coronal and Sagittal MRI in Temporomandibular Joint Disorders. Clinical Oral Investigations, 17, 1243-1250.

https://doi.org/10.1007/s00784-012-0803-4

[15] Dias, I.M., Cordeiro, P.C., Devito, K.L., Tavares, M.L., Leite, I.C., et al. (2016) Evaluation of Temporomandibular Joint Disc Displacement as a Risk Factor for Osteoarthrosis. Journal of Oral and Maxillofacial Surgery, 45, 313-317.

https://doi.org/10.1016/j.ijom.2015.09.016

[16] Hamada, Y., Kondoh, T., Holmlund, A.B., Yamamoto, M., Horie, A., et al. (2006) Inflammatory Cytokines Correlated with Clinical Outcome of Temporomandibular Joint Irrigation in Patients with Chronic Closed Lock. Oral Surgery, Oral Medicine, Oral Pathology, Oral Radiology, and Endodontics, 102, 596-601.

https://doi.org/10.1016/j.tripleo.2005.11.010

[17] Shibata, T., Murakami, K., Kubota, E. and Maeda, H. (1998) Glycosaminoglycan Components in Temporomandibular Joint Synovial Fluid as Markers of Joint Pathology. Journal of Oral and Maxillofacial Surgery, 56, 209-213. https://doi.org/10.1016/S0278-2391(98)90871-0

[18] Roh, H.S., Kim, W., Kim, Y.K. and Lee, J.Y. (2012) Relationships between Disk Displacement, Joint Effusion and Degenerative Changes of the TMJ in TMD Patients Based on MRI Findings. Journal of Cranio-Maxillofacial Surgery, 40, 283-286. https://doi.org/10.1016/j.jcms.2011.04.006

[19] Alves, B.M., Macedo, C.R., Januzzi, E., Grossmann, E., Atallah, Á.N., et al. (2013) Mandibular Manipulation for the Treatment of Temporomandibular Disorder. Journal of Craniofacial Surgery, 24, 488-493. https://doi.org/10.1097/SCS.0b013e31827c81b3

[20] Al-Baghdadi, M., Durham, J., Araujo-Soares, V., Robalino, S., Errington, L., et al. (2014) TMJ Disc Displacement without Reduction Management: A Systematic Review. Journal of Dental Research, 93, 37S-51S. https://doi.org/10.1177/0022034514528333

[21] Schiffman, E.L., Velly, A.M., Look, J.O., Hodges, J.S., Swift, J.Q., et al. (2014) Effects of Four Treatment Strategies for Temporomandibular Joint Closed Lock. Journal of Oral and Maxillofacial Surgery, 43, 217-226 https://doi.org/10.1016/j.ijom.2013.07.744

[22] Seedorf, H., Scholz, A., Kirsch, I., Fenske, C. and Jüde, H.D. (2007) Pivot Appliances-Is There a Distractive Effect on the Temporomandibular Joint? Journal of Oral Rehabilitation, 34, 34-40. https://doi.org/10.1111/j.1365-2842.2005.01557.x

[23] Yıldız, M., Çağatay, D.S., Şakar, O. and Sülün, T. (2018) Distraction of the Temporomandibular Joint Condyle in Patients with Unilateral Non-Reducing Disc Displacement: Fact or Fiction? Cranio, 36, 294-299. https://doi.org/10.1080/08869634.2017.1350337

[24] Sembronio, S., Albiero, A.M., Toro, C., Robiony, M. and Politi, M. (2008) Is There a Role for Arthrocentesis in Recapturing the Displaced Disc in Patients with Closed Lock of the Temporomandibular Joint? Oral Surgery, Oral Medicine, Oral Pathology and Oral Radiology, 105, 274-280. https://doi.org/10.1016/j.tripleo.2007.07.003

[25] Yoshida, H., Sakata, T., Hayashi, T., Shirao, K., Oshiro, N., et al. (2011) Evaluation of Mandibular Condylar Movement Exercise for Patients with Internal Derangement of the Temporomandibular Joint on Initial Presentation. British Journal of Oral and Maxillofacial Surgery, 49, 310-313. https://doi.org/10.1016/j.bjoms.2010.05.016

[26] Haketa, T., Kino, K., Sugisaki, M., Takaoka, M. and Ohta, T. (2010) Randomized Clinical Trial of Treatment for TMJ Disc Displacement. Journal of Dental Research, 89, 1259-1263. https://doi.org/10.1177/0022034510378424

[27] Major, P.W., Kinniburgh, R.D., Nebbe, B., Prasad, N.G. and Glover, K.E. (2002) Tomo- 
graphic Assessment of Temporomandibular Joint Osseous Articular Surface Contour and Spatial Relationships Associated with Disc Displacement and Disc Length. American Journal of Orthodontics and Dentofacial Orthopedics, 121, 152-161. https://doi.org/10.1067/mod.2002.120641

[28] Larheim, T.A., Hol, C., Ottersen, M.K., Mork-Knutsen, B.B. and Arvidsson, L.Z. (2018) The Role of Imaging in the Diagnosis of Temporomandibular Joint Pathology. Oral and Maxillofacial Surgery Clinics of North America, 30, 239-249.

https://doi.org/10.1016/j.coms.2018.04.001

[29] Oğütcen-Toller, M., Taşkaya-Yilmaz, N. and Yilmaz, F. (2002) The Evaluation of Temporomandibular Joint Disc Position in TMJ Disorders Using MRI. Journal of Oral and Maxillofacial Surgery, 31, 603-607. https://doi.org/10.1054/ijom.2002.0321

[30] Larheim, T.A., Westesson, P. and Sano, T. (2001) Temporomandibular Joint Disk Displacement: Comparison in Asymptomatic Volunteers and Patients. Radiology, 218, 428-432. https://doi.org/10.1148/radiology.218.2.r01fe11428

[31] Ikeda, R. and Ikeda, K. (2015) Directional Characteristics of Incipient Temporomandibular Joint Disc Displacements: A Magnetic Resonance Imaging Study. American Journal of Orthodontics and Dentofacial Orthopedics, 49, 39-45. https://doi.org/10.1016/j.ajodo.2015.06.021

[32] Sener, S. and Akgänlü, F. (2004) MRI Characteristics of Anterior Disc Displacement with and without Reduction. Dentomaxillofacial Radiology, 33, 245-152.

https://doi.org/10.1259/dmfr/17738454

[33] Tasaki, M.M., Westesson, P.L., Isberg, A.M., Ren, Y.F. and Tallents, R.H. (1996) Classification and Prevalence of Temporomandibular Joint Disk Displacement in Patients and Symptom-Free Volunteers. American Journal of Orthodontics and Dentofacial Orthopedics, 109, 249-262. https://doi.org/10.1016/S0889-5406(96)70148-8

[34] Uchida, T., Komiyama, O., Okamoto, Y., Iida, T., Wakami, M., et al. (2015) Evaluation of Temporomandibular Joint Disk Displacement in Asymptomatic Volunteers Using Magnetic Resonance Imaging. International Journal of Oral Science, 14, 21-27. https://doi.org/10.5466/ijoms.14.21

[35] Takahara, N., Imai, H., Nakagawa, S., Sumikura, K., Tsushima, F., et al. (2014) Temporomandibular Joint Intermittent Closed Lock: Clinic and Magnetic Resonance Imaging Findings. Oral Surgery, Oral Medicine, Oral Pathology, Oral Radiology, 118, 418-423. https://doi.org/10.1016/j.oooo.2014.05.019 